\title{
Comment on "Exact bosonization for an interacting Fermi gas in arbitrary dimensions"
}

\author{
D. Galanakis, ${ }^{1}$ S. Yang, ${ }^{1}$ F.F. Assaad, ${ }^{2}$ M. Jarrell,${ }^{1}$ P. Werner,${ }^{3}$ and M. Troyer ${ }^{3}$ \\ ${ }^{1}$ Department of Physics and Astronomy, Baton Rouge, LA, USA \\ ${ }^{2}$ Institut für theoretische Physik und Astrophysik, \\ Universität Würzburg, Am Hubland, D-97074, Germany \\ ${ }^{3}$ Theoretische Physik, ETH Zurich, 8093, Zurich, Switzerland
}

In a recent Letter [1] Efetov et al. propose an exact mapping of an interacting fermion system onto a new model that is supposed to allow sign-problem free Monte Carlo simulations. In this Comment, we show that their formalism is equivalent to the standard approach of Blackenbecker, Scalapino and Sugar (BSS) 2] for fermionic systems and has the same sign statistics and minus sign problem.

Our first observation is that the partition function for a given configuration of the auxiliary fields $\phi$ is the same in the standard formulation $Z_{f}$ [Eq. (8) in Ref. [1]] and in their new bosonized scheme $Z_{b}$ [Eq. (9)]:

$$
Z_{f}[\phi]=Z_{b}[\phi] .
$$

This observation is trivial in the limit of the time step $\Delta \tau \rightarrow 0$, where both schemes reproduce the same partition function. Since $Z_{f}$ can be negative also in this limit [2], $Z_{b}$ is also not sign-positive. Both $Z_{f}$ and $Z_{b}$ are positive if $\phi$ is a smooth path [2], but restricting the configuration space to smooth paths amounts to a semiclassical approximation.

We next show that $Z_{f}[\phi]=Z_{b}[\phi]$ also holds for finite $\Delta \tau$ and piecewise constant paths where the field $\phi_{r}(\tau)$ is constant on the interval $[(l+1) \Delta, l \Delta]$. Efetov et al.'s Eq. (9) is equivalent to $Z_{b}[\phi]=\operatorname{Tr}\left(e^{-\beta H_{0}} U_{I}(\beta, 0)\right)$ where $\frac{\partial}{\partial \tau_{1}} U_{I}\left(\tau_{1}, \tau_{0}\right)=-H_{I, 1}\left(\tau_{1}\right) U_{I}\left(\tau_{1}, \tau_{0}\right)$ and $H_{I, 1}(\tau)=-\sum_{r} \phi_{r}(\tau) e^{\tau H_{0}} m_{z, r} e^{-\tau H_{0}}$. Since $U_{I}\left(\tau_{2}, \tau_{0}\right)=U_{I}\left(\tau_{2}, \tau_{1}\right) U_{I}\left(\tau_{1}, \tau_{0}\right), \quad Z_{b}[\phi]=$ $\operatorname{Tr}\left[e^{-\beta H_{0}} \prod_{n=0}^{N} U_{I}(l \Delta,(l-1) \Delta)\right] . \quad$ We are left with the task of evaluating $U_{I}(l \Delta,(l-1) \Delta)$ on the $l^{\text {th }}$ time interval where the fields are constant and take the values $\phi_{r l}$. For this constant in time field configuration, $U_{I}(l \Delta,(l-1) \Delta)=e^{l \Delta H_{0}} e^{-\Delta h_{l}} e^{-(l-1) \Delta H_{0}}$ with $H_{l}=H_{0}-\sum_{r} \phi_{r, l} m_{z, r}$. Hence,

$$
Z_{b}[\phi]=\operatorname{det}\left[1+\prod_{l=0}^{N} e^{-\Delta h_{l}}\right] \equiv Z_{f}[\phi] .
$$

We also demonstrate that the proposed formalism is equivalent to that of BSS [2]. Starting from the expression for $Z$ found between Eq. (9) and Eq. (10) and using a matrix notation the partition function reads

$$
\ln \frac{Z_{b}[\phi]}{Z_{0}}=\int_{0}^{1} d u \sum_{\sigma} \operatorname{Tr} \Phi G_{\sigma}
$$

where $\Phi$ is a matrix with elements $\Phi\left(r l, r^{\prime} l^{\prime}\right)=$ $\phi_{r, l} \delta_{r r^{\prime}} \delta_{l l^{\prime}}$, the trace is over the space time indices and

$$
G_{\sigma}=G^{0}+u \sigma G^{0} \Phi G_{\sigma}=\left(1-u \sigma G^{0} \Phi\right)^{-1} G^{0},
$$

where $G^{0}$ is the non interacting Green function. From Eqs. (3) and (4) we get

$$
\ln \frac{Z_{b}[\phi]}{Z_{0}}=\int_{0}^{1} d u \sum_{\sigma} \operatorname{Tr} \frac{1}{1-u \sigma G^{0} \Phi} \sigma G^{0} \Phi .
$$

The integral in terms of $u$ and $\sigma$ can be carried out analytically yielding the BSS partition function

$$
\begin{aligned}
Z_{b}[\phi] & =Z_{0} \exp \left(-\operatorname{Tr}\left[\ln \left(1-\left(G^{0} \Phi\right)^{2}\right)\right]\right) \\
& =\frac{Z_{0}}{\operatorname{det}\left(1-\left(G^{0} \Phi\right)^{2}\right)}=\prod_{\sigma=\uparrow \downarrow} \frac{1}{\operatorname{det} G_{\sigma}} .
\end{aligned}
$$

A sign problem is present in Eq. (3), but is hidden in the $u$ integral. If $G^{0} \Phi$ has eigenvalues on the real axis with absolute value greater than 1 , then the $u$ integral runs over poles. This will give phases of $i \pi$ in the exponent which can lead to negative values of the exponential.

Finally, Efetov's et al. suggest an alternative way of evaluating the same weight $Z_{b}$ from a bosonic field $A_{r r^{\prime}}(\tau)$; however, the equation of motion that $A$ satisfies, [their Eq. (13)], is singular. Furthermore, the equation of motion cannot uniquely determine $A$ - not even with their additional constraint $\sum_{r} A_{r r}(\tau)=0$. The latter can be shown, for example, using a two site cluster and a single time slice.

In conclusion, we have shown that for piecewise continuous paths the partition function obtained from Efetov et al.'s method [1] is equivalent to the standard BSS formulation and has, in particular, the same sign problem. However, their method provides a new perspective on the minus sign problem, as it can be viewed as originating from poles of the coupling constant $(u)$ integral, or as branch cuts of a logarithm when the integral over $u$ is performed analytically.

[1] K. B. Efetov, C. Pépin, and H. Meier, Phys. Rev. Lett. 103, 186403 (2009).

[2] R. Blankenbeckler, D. J. Scalapino, and R. L. Sugar, Phys. Rev. D 24, 2278 (1981). 